\title{
Data Hiding Using Sequential Hamming + k with m Overlapped Pixels
}

\author{
Cheonshik Kim $^{1 *}$, Dongkyoo Shin ${ }^{1 *}$, Ching-Nung Yang ${ }^{2}$, Yi-Cheng Chen ${ }^{2}$, Song-Yu Wu ${ }^{2}$ \\ ${ }^{1}$ Dept. of Computer Science and Engineering, Sejong University, \\ Seoul, Republic of Korea \\ [e-mail: mipsan@paran.com, shindk@sejong.ac.kr] \\ ${ }^{2}$ Dept. of Computer Science and Information Engineering, \\ National Dong Hwa University, Hualien, Taiwan \\ [e-mail: cnyang@gms.ndhu.edu.tw, hippo032318@gmail.com, \\ 610621220@gms.ndhu.edu.tw] \\ *Corresponding author: D. Shin, C. Kim
}

Received November 7, 2018; revised January 22, 2019; accepted June 13, 2019;

published December 31, 2019

\begin{abstract}
Recently, Kim et al. introduced the Hamming $+k$ with $m$ overlapped pixels data hiding ( $\mathrm{H}_{-}$ $m \mathrm{DH}$ ) based on matrix encoding. The embedding rate (ER) of this method is 0.54 , which is better than Hamming code HC $(n, n-k)$ and HC $(n, n-k)+1 \mathrm{DH}(\mathrm{H} 1 \mathrm{DH})$, but not enough. Hamming code data hiding (HDH) is using a covering function $\operatorname{COV}\left(1, n=2^{k}-1, k\right)$ and $\mathrm{H} 1 \mathrm{DH}$ has a better embedding efficiency, when compared with HDH. The demerit of this method is that they do not exploit their space of pixels enough to increase ER. In this paper, we increase ER using sequential $\mathrm{H} k \_m \mathrm{DH}\left(\mathrm{SH} k \_m \mathrm{DH}\right)$ through fully exploiting every pixel in a cover image. In SH $k \_m D H$, a collision maybe happens when the position of two pixels within overlapped two blocks is the same. To solve the collision problem, in this paper, we have devised that the number of modification does not exceed 2 bits even if a collision occurs by using OPAP and LSB. Theoretical estimations of the average mean square error (AMSE) for these schemes demonstrate the advantage of our SH $k$ $m \mathrm{DH}$ scheme. Experimental results show that the proposed method is superior to previous schemes.
\end{abstract}

Keywords: Data Hiding, Matrix Encoding, Hamming DH (HDH), Hamming+1 DH (H1DH), $\mathrm{H} k \_m \mathrm{DH}$ 


\section{Introduction}

Secure communication is often accomplished through cryptography. However, For secret communication, steganography is more suitable than cryptography. The reason is the purpose of steganography is that the existence of hidden data is not detected by the adversary [1]. This technique uses a communication channel to transmit secretly after concealing messages on various media and it is called data hiding (DH) [2]. DH is to embed secret bits by slightly modifying the cover media. In fact, images contain a lot of redundant bits, making them very suitable for delivering secret messages. Meanwhile, techniques for detecting hidden data in images, i.e., steganalysis [3, 4], have also been actively researched. Therefore, most researches on DH have been dedicated on minimizing the damage of an image while allowing sufficient messages to be concealed in images.

The method based on Least Significant Bit (LSB) is easily implemented by increasing or decreasing the values of pixels by only one to match the pixels of the image with the bits of the data. Meanwhile, in the aspect of image quality based on LSB, the optimal pixel adjustment process (OPAP) [5] is a great help for improving peak signal to noise ratio (PSNR) of stego image. Using only LSB, it is impossible to guarantee the quality of a stego image, because it could provoke distortion. Recently, Yang et al. [6] adopted LSB and OPAP with a cover image generated neighbor mean interpolation to improve visual quality in $\mathrm{DH}$.

Matrix encoding is a method to embed or extract the message by using syndrome. Crandall [7] proposed a DH called matrix encoding, Westfeld implemented the F5 algorithm [8] using Hamming code, and Bierbrauer et al. [9] analyzed the possibility of this method. Willems [10] introduced a binary embedding method and a ternary embedding method using Hamming code and Golay code respectively. The F5 algorithm is a method that conceals $k$ bits into $\left(2^{k}-1\right)$ pixels by using modifying the only one bit. F5 has higher embedding efficiency (EE) (which is the average number of embedded bits per change) [11] than the simple LSB method. Since then, many kinds of research based on matrix encoding have been developed inspired by the F5 [12].

Zhang [13] and Fridrich [14] proposed ternary Hamming code that each secret digit in a $(2 n+1)$-ary notational system is carried by $n$ cover pixels by increasing or decreasing only one bit. Mielikainen [15] devised LSB matching revised method to achieve the same payload by modifying fewer pixels to the cover image than a simple LSB-based method. In Mielikainen's scheme, the embedding procedure performs for two cover pixels, $x_{i}$ and $x_{i}+1$, at a time. The value of the $i^{\text {th }}$ message bit $m_{i}$ is equal to the LSB of the $i^{\text {th }}$ pixel $y_{i}$. The value of the $i+1^{\text {th }}$ message bit $m_{i}+1$ is a function (LSB $\left(\left\lfloor y_{i} / 2\right\rfloor+y_{i}+1\right)$ ) of $y_{i}$ and $y_{i}+1$. Zhang et al. [11] proposed Hamming $+1 \mathrm{DH}(\mathrm{H} 1 \mathrm{DH})$ embedding one more bit than COV $\left(1,2^{k}-1, k\right)$ with using one additional pixel. In H1DH, the performance improves by employing the OPAP. Recently, Kim and Yang [16] proposed a method of overlapping three pixels to improve ER with $\operatorname{COV}\left(1,2^{k}-1, k\right)$, and the ER was increased to about 0.12 compared to HDH.

In [16], the second covering function $\operatorname{COV}\left(1,2^{k}-1, k\right)$ uses a second LSB. Therefore, it causes a high distortion. Hamming $+\mathrm{k}(\mathrm{H} k \mathrm{DH})$ [17] is a method to embed $2 k$ in a fully overlapped virtual block (LSB $\oplus$ 2LSB) by using COV $\left(1,2^{k}-1, k\right)$, and OPAP and LSB were used for optimization. Therefore, $\mathrm{H} k \mathrm{DH}$ has achieved high ER.

Afterward, Kim et al. [18] extended $\mathrm{H} k \mathrm{DH}$ to $m$ overlapped pixels data hiding (Hk_mDH) to embed $2 k$ bits in $\left(2^{k+1}-m-2\right)$ pixels by modifying at most 3 bits. But, if the values of two $\operatorname{COV}\left(1,2^{k}-1, k\right)$ are the same, a collision problem may occur, which may inevitably cause a mean square error. Finally, the $\mathrm{H} k \_m \mathrm{mH}$ achieves better embedding rate compared with 
$\mathrm{H} k \mathrm{DH}$. The problem of $\mathrm{H} k \_m \mathrm{mH}$ is that the number of pixels that need to be excavated to embed many bits still remains. In this paper, we introduce sequential $\mathrm{H} k \_m \mathrm{mH}$ (SH $\left.k \_m \mathrm{DH}\right)$ to solve the matter of $H k \_m D H$. SH $k \_m D H$ has a way of improving the performance of the ER without significantly degrading the quality of the cover image.

The rest of this paper is organized as follows. Section 2 concisely reviews Hamming code, $\mathrm{HDH}$, and Kim and Yang's DH, and Hk_mDH. In Section 3, we present SHk_mDH. In addition, the AMSE of SHk $m \mathrm{mDH}$ is theoretically derived. In Section 4, the proposed SHk $m$ DDH is tested using some images. A comparison with the HDH, H1DH, Kim and Yang's DH, and Hk_mDH is also provided. In addition, we apply RS steganlysis to SHk_mDH. Finally, this paper concludes in Section 5.

\section{Related Work}

\subsection{Hamming Code}

$\mathrm{HC}(n, k)$ codes are a single error correction linear code with $\mathrm{d}_{\min }=3$ and parity check matrix $\mathbf{H}$ of $k \times n\left(=2^{k}-1\right)$, where $k$ is the number of information bits and $(n-k)$ is the number of parity bits. The parity check matrix $\mathbf{H}$ for $k=3$ is

$$
\mathbf{H}=\left[\begin{array}{lllllll}
0 & 0 & 0 & 1 & 1 & 1 & 1 \\
0 & 1 & 1 & 0 & 0 & 1 & 1 \\
1 & 0 & 1 & 0 & 1 & 0 & 1
\end{array}\right]
$$

The $n$-bit column vectors $x_{b}$ and $y_{b}$ are LSB of pixels $x=\left(x_{1}, \ldots, x_{n-k}\right)$ and $y=$ $\left(y_{1}, \ldots, y_{n}\right)$, where $x_{b}$ and $y_{b}$ will be denoted $F_{2}^{n-k}$ and $F_{2}^{n}$, respectively.

Let $y_{b}$ is obtained from an information bits $x_{b}$ via $(n-k) \times n$ generator matrix $G$, i.e., $y_{b}=x_{b} \cdot G$. For any $y_{b}$, the vector $S=H y_{b}{ }^{T}\left(\in F_{2}^{k}\right)$ is called the syndrome of $y_{b}$. For each syndrome $S$, the set $\hat{C}(S)=\left\{y_{b} \in F_{2}^{n} \mid \mathrm{H} y_{b}=S\right\}$ is called a coset. Note that $\hat{C}(0)=\hat{C}$. For any syndrome $S$, let $\operatorname{dec}(\mathrm{S})$ be the integer. For any non-zero syndrome $\mathrm{S}$, the vector $e(S)=$ $(0, \ldots, 1, \ldots, 0)$ with 1 at the $\operatorname{dec}(S)$-th place, i.e., $\mathrm{He}(S)=S$. To embed $\delta\left(\in F_{2}^{k}\right)$ message bits, replace $y$ with $\hat{y}=y_{b} \oplus e\left(\delta-\mathrm{H} y_{b}\right)$. The receiver extracts the message from $\hat{y}$ by Eq. (2).

$$
\left\{\begin{array}{c}
\hat{y} \cdot H^{T}=\left(y_{b} \oplus e\right) \cdot H^{T}=y_{b} \cdot H^{T}+\delta-y_{b} \cdot H^{T} \\
=\delta
\end{array}\right.
$$

In order to embed $\delta$ message bits in each subset by making at most one embedding change, we divide the cover image into $N / n\left(=2^{k}-1\right)$ subsets, each consisting of $n$ pixels. Suppose that there is one error bit occurred in $\hat{y}$ (say the 6-th bit from left), i.e., $e=\left(e_{1}, \ldots, e_{7}\right)=$ (0000010). The syndrome $S=(110)$ is extracted. It means that the error position is the 6-th from left.

\subsection{Hamming coding+1 data hiding (H1DH)}

For H1DH, $k$ secret bits $\delta$ embed to $y_{b}=\left(y_{1}, \ldots, y_{n}\right)$ of $n\left(=2^{k}-1\right)$ pixels using $\operatorname{COV}(1, n, k)$ by flipping at most one bit. An extra 1-bit embeds at $2^{k}$ by using OPAP. That is, first $k$ was embedded by using syndrome $\hat{y}=y_{b} \oplus e\left(\delta-\mathrm{Hy}_{b}\right)$. If $\left(\hat{y} \cdot H^{T}=\delta\right)$, there is no needed any action. For embedding $\delta$, one change is needed with probability $\left(2^{k}-1\right) / 2^{k}$. To embed $k+1$ bits, they append one pixel like $y=\left(y_{1}, \ldots, y_{n}, y_{n+1}\right)$. By $y_{i} \pm 1$, its $b\left(y_{i}\right)$ 
becomes the same binary $b\left(y_{i}\right) \oplus 1$, while $2 b\left(y_{i}\right)$ can either be 0 or 1 after $b\left(y_{i}\right) \oplus 1$, where $b(\cdot)$ $=\left(y_{i} \bmod 2\right)$ and $2 b(\cdot)=\left(\left\lfloor y_{i} / 2\right\rfloor \bmod 2\right)$ are LSB and 2LSB, respectively.

$$
\begin{gathered}
\left(\delta_{1}, \ldots, \delta_{k}\right)^{T}=H \cdot y_{b} \\
\delta_{k+1}=\left(2 b\left(y_{1}\right)+\cdots+2 b\left(y_{2^{k}-1}\right)+b\left(y_{2^{k}}\right)\right) \bmod 2
\end{gathered}
$$

There are four embedding optimal rules for H1DH.

(O1): When Eqs. (3) and (4) are satisfied, no action.

(O2): When only Eq. (3) is fulfilled, process $y_{2^{k}} \pm 1$.

(O3): When only Eq. (4) is satisfied, process $y_{i} \pm 1$.

(O4): When both of two are not satisfied, process $y_{2^{k}} \pm 1$.

\subsection{Hamming+ $k$ scheme with $m$ overlapped pixels $\left(\mathrm{H} k \_m D H\right)$}

The Hk_mDH [19] is the extended method of $\mathrm{H} k \mathrm{DH}$ [18]. Suppose that one block is composed of $\left(2^{k+1}-m-2\right)$ pixels $\left(y_{1}, \ldots, y_{2^{k+1}-m-2}\right)$ with $m$ overlapped pixel.

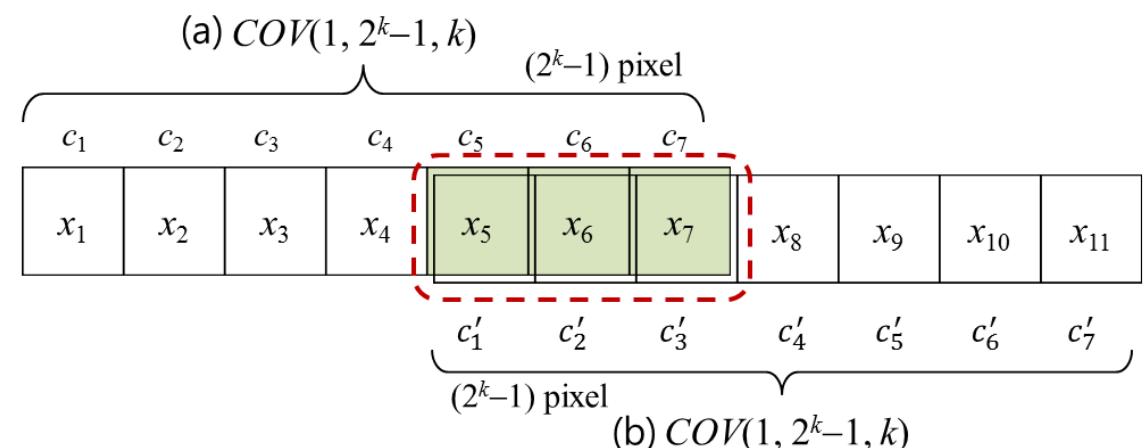

Fig. 1. Block diagram of $\mathrm{H} k \_m \mathrm{DH}$ with $k=3$ and $m=3$.

Hk_mDH uses $\operatorname{COV}\left(1,2^{k}-1, k\right)$ to embed $k$ secret bits $\left(\delta_{1}, \ldots, \delta_{k}\right)$ into the first $\left(2^{k}-1\right)$ pixels, and then embed the other $k$ secret bits into the last $\left(2^{k}-1\right)$ pixels. The first $\left(2^{k}-1\right)$ pixels are $\left(y_{1}, \ldots, y_{2^{k}-1}\right)$, and the other pixels are $\left(y_{2^{k}-m}, \ldots, y_{2^{k}-m-1}\right)$. As shown in Fig. 1, consider the example $k=3$ (i.e., using $(7,4)$ Hamming code) and $m=3$. There are total $11\left(=2^{3+1}-3-2\right)$ pixels with 3 overlapped pixels. To embed secret bits, we apply Eqs. (5) and (6) to each pixels $\left(2^{k}-1\right)$.

$$
\begin{aligned}
& \left\{\begin{array}{c}
p=\left(\left(b\left(y_{1}\right), \ldots, b\left(y_{2^{k}-m-1}\right), b\left(y_{2^{k}-m}\right) \oplus 2 b\left(y_{2^{k}-m}\right), \ldots, b\left(y_{2^{k}-1}\right) \oplus 2 b\left(y_{2^{k}-1}\right)\right)\right. \\
\left(\delta_{1}, \ldots, \delta_{k}\right)^{T}=H \cdot p^{T}
\end{array}\right. \\
& \left\{\begin{array}{c}
q=\left(\left(2 b\left(y_{2^{k}-m}\right), \ldots, 2 b\left(y_{2^{k}-1}\right),\left(y_{2^{k}}\right), \ldots, b\left(y_{2^{k+1}-m-2}\right)\right)\right. \\
\left(\delta_{k+1}, \ldots, \delta_{2 k}\right)^{T}=H \cdot q^{T}
\end{array}\right.
\end{aligned}
$$

We embed $k$ secret bits into the first seven bits $p$ for Eq. (5), and then embed the other $k$ secret bits into the other seven bits $q$ of Eq. (6). Except the $m$ overlapped pixels, other pixels adopt the LSBs. The overlapped pixels in Eq. (5) (respectively, Eq. (6)), are the XOR-ed 
results of $1^{\text {st }} \mathrm{LSB}$ and $2^{\text {nd }} \mathrm{LSB}$, and $2^{\text {nd }} \mathrm{LSB}$, respectively.

The embedding algorithm is as follows.

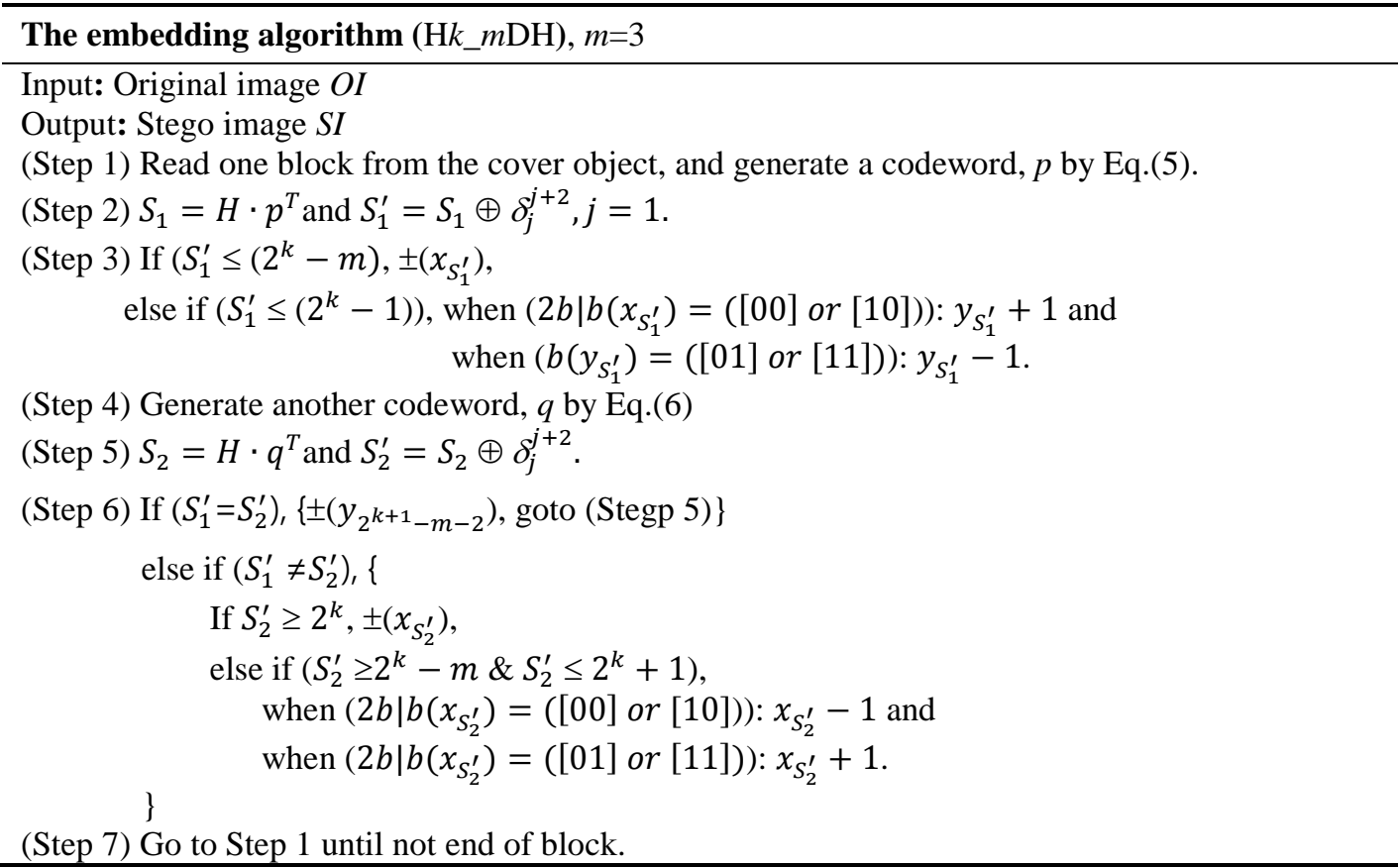

\section{The Proposed Schemes}

\subsection{Design concept}

All Hamming code based DHs are shown in Fig. 2. The ERs for HDH, H1DH, HkDH, and H $\_\_m D H$ are $k / n,(k+1) / n, 2 k / n, 2 k /(2 n-m)$, respectively (see Figs. 2(a) (d)). As shown in Fig. 2(e), the proposed SH $\_m D H$ is to sequentially use Hk $\_m D H$ to embed secret bits. The ER of the proposed SH $\_m D H$ is derived as Eq. (7).

$$
E R=\frac{\alpha \cdot k}{\alpha \cdot(n-m)+m}=\frac{k}{(n-m)+m / \alpha}=\frac{k}{n-m}(\text { for } \alpha \rightarrow \infty)
$$

The value of $k /(n-m)$ is larger than $k /(2 n-m)$ of $H k \_m D H$. For instance, when $\alpha=3, n=7$, and

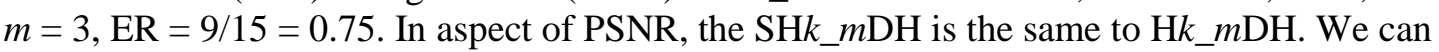
reduce the distortion by using OPAP and LSB.

In our proposed block overlapping approach, an image is divided by blocks of sized $(1 \times n)$ pixels. At this time, every block has $2 m$ overlapped pixels except for the first block including $m$ overlapped pixels. The main difference between the SH $k$ mDH and the $\mathrm{H} k \_m D H$ is that we sequentially embed secret bits with $(n-2 m) \geq 0$ (see Fig. 2(e)). 

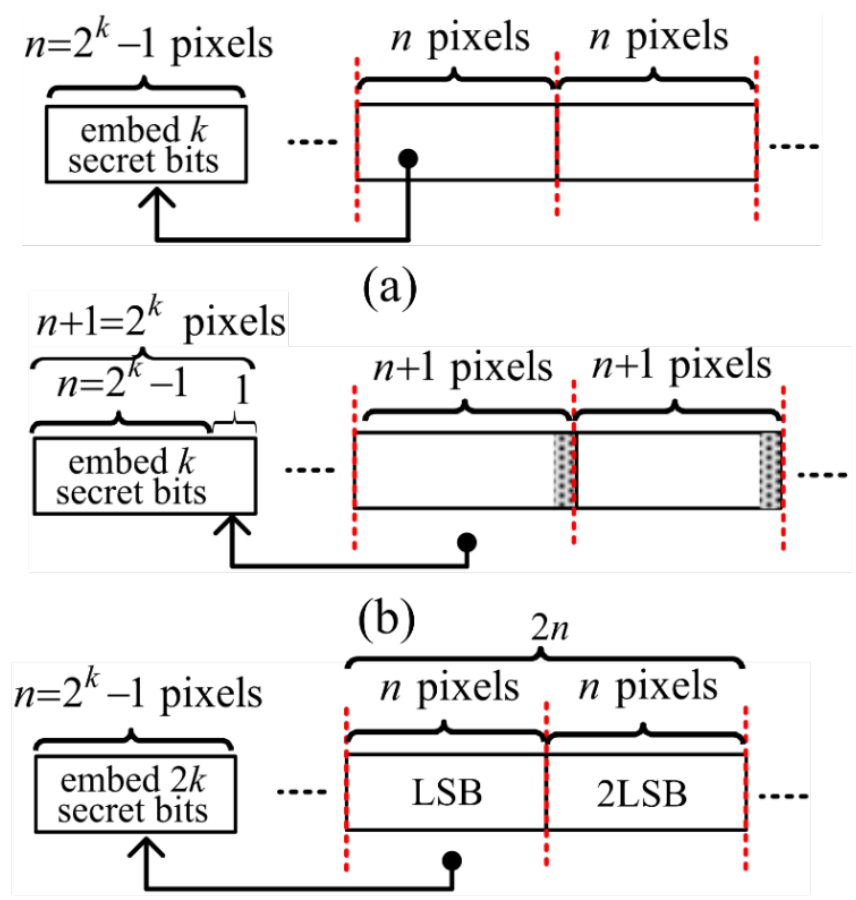

(c)

$2 n-m=2^{k+1}-m-2$ pixels

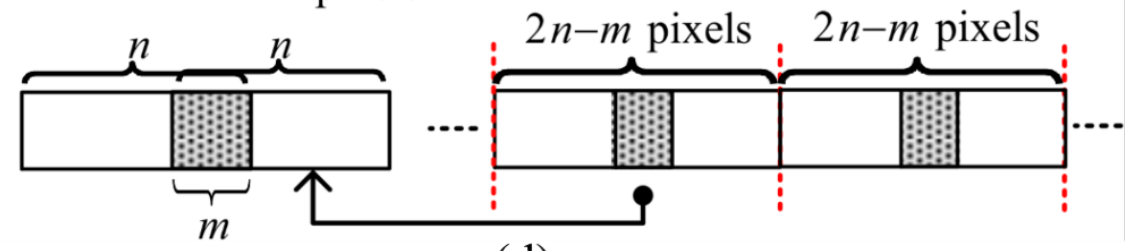

(d)

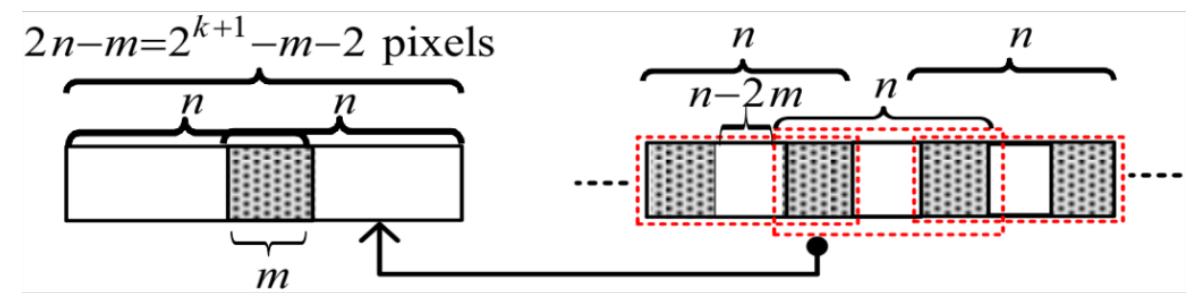

(e)

Fig. 2. Diagrammatical representation of Hamming code based DH:

(a) $\mathrm{HDH}$ (b) $\mathrm{H} 1 \mathrm{DH}$ (c) $\mathrm{H} k \mathrm{DH}$ (d) $\mathrm{H} k \_m \mathrm{mH}$ (e) the SHk_mDH.

\subsection{Embedding Procedure}

For brief explanation, we here assume that $k=3$ (i.e., by $\operatorname{COV}(1,7,3)$ covering function) and $m=3$ for the proposed SH $k m D H$, which can embed 3 secret bits into 7 overlapped cover pixels.

The detailed procedure of the embedding is as follow. 


\section{The embedding algorithm $\left(\mathrm{SH} k \_m \mathrm{DH}\right)$}

Input: Original image OI

Output: Stego image SI

(Step 1) Read one block, overlapped $\left(2^{k}-1\right)$ pixels $\left(x_{i}^{2^{k}-1}\right)$, from the cover object and generate a codeword, $p \in F_{2}^{n}$, by Eq.(8).

(Step 2) Calculate the syndrome $S=H \bullet p^{T}$ and $S^{\prime}=S \oplus\left(\delta_{j=1}^{j+2}\right)$.

(Step 3) if $S \neq 0$ \{if (S $\leq 4)$ \{figure out Eq. (9) \},

else if $(S \leq 7)\{$ figure out Eq. (10)\}.

Note: $2 \mathrm{~b} \mid \mathrm{b}(\boldsymbol{x})$ means 2LSB and LSB of parameter pixel $x$ in Eqs.(9) and (10).

(Step 4) If not end of the block, $i=i+4, j=j+3$ and go to Step 1 .

$$
\begin{gathered}
p=\left(b\left(x_{1}\right) \oplus b\left(\left\lfloor\frac{x_{1}}{2}\right\rfloor\right), \ldots, b\left(x_{4}\right) \oplus\left(\left\lfloor\frac{x_{4}}{2}\right\rfloor\right), b\left(\left\lfloor\frac{x_{5}}{2}\right\rfloor\right), \ldots, b\left(\left\lfloor\frac{x_{7}}{2}\right\rfloor\right)\right) \\
x_{S^{\prime}}= \begin{cases}x_{S^{\prime}}+1 & \text { if }\left(2 b \mid b\left(x_{S^{\prime}}\right)=([00] \text { or }[10])\right) \\
x_{S^{\prime}}-1 & \text { if }\left(2 b \mid b\left(x_{S^{\prime}}\right)=([01] \text { or }[11])\right)\end{cases} \\
x_{S^{\prime}}= \begin{cases}x_{S^{\prime}}+1 & \text { if }\left(2 b \mid b\left(x_{S^{\prime}}\right)=([01] \text { or }[11])\right) \\
x_{S^{\prime}}-1 & \text { if }\left(2 b \mid b\left(x_{S^{\prime}}\right)=([00] \text { or }[10])\right)\end{cases}
\end{gathered}
$$

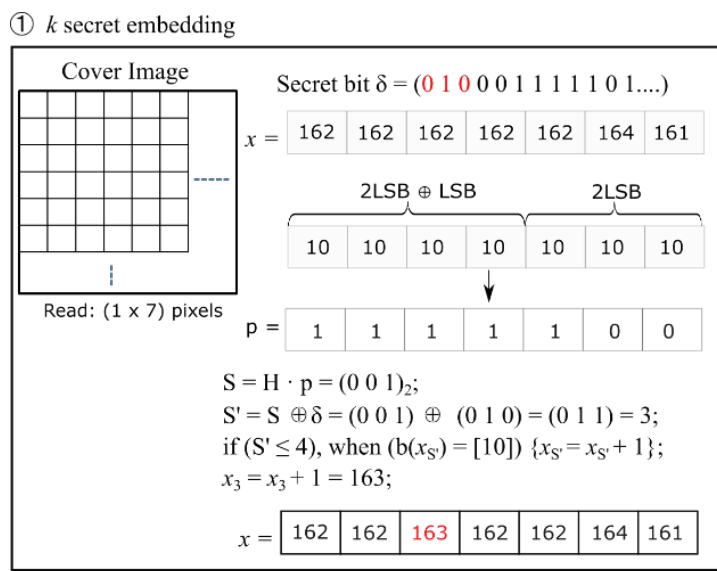

(2) $k+3$ secret embedding

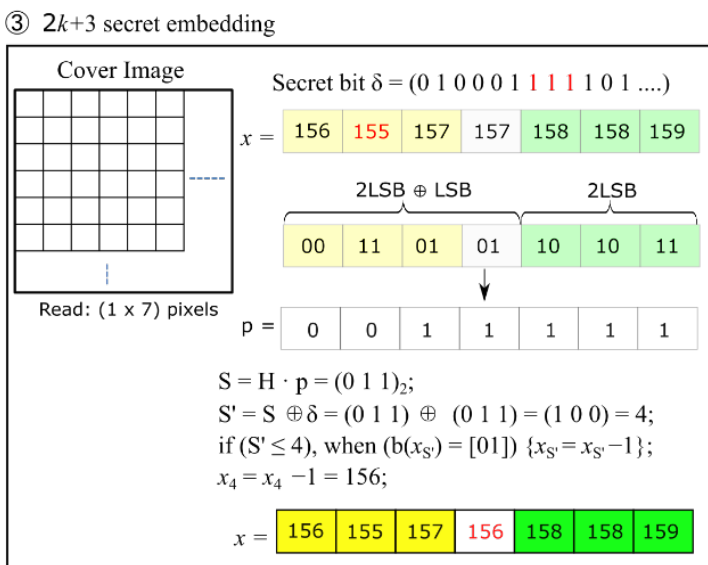

(4) $3 k+3$ secret embedding

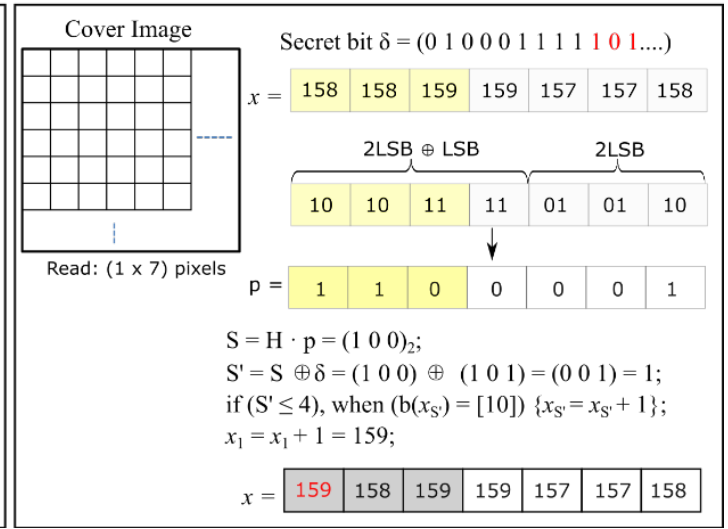

Fig. 3. Embedding example using $\mathrm{SH} k \_m \mathrm{mH}$. 
Example 1: We demonstrate the embedding procedure using given $x$ and $\delta$ as shown in Fig. 3 . For embedding, first, $x=(162,162,162,162,162,164,161)$ and $\delta=(010)$ is given.

The codeword $p$ is obtained from a vector $x$ using Eq. (8) and then figure out syndrome $\mathrm{S}=$ $\mathrm{H} \cdot p=(001)$ using the codeword $p$. First 4 bits of the codeword is obtained by using 2LSB $\oplus$ LSB. The other 3-bits are extracted from 2LSB of the $x$ directly. $S^{\prime}(=S \oplus \delta)$ means the codeword $p$ has new error $e=(0010000)$. If we flip the third bit, then syndrome of the codeword $p$ ' is equal to $\mathrm{S}=(011)$. However, it is not acceptable to modify a bit in the codeword $p$ for embedding secret bits directly, because the codeword $p$ cannot store any bits. Therefore, we must modify a pixel in the vector $x$ directly according to the rule in Eqs. (9) and (10). In this case, since $S^{\prime}$ is 3 , it is belonging to the rule of Eq. (9). Thus, because $b 2 \mid b\left(x_{S^{\prime}}\right)$ is equal to "[10]," figure out $x_{\mathrm{S}^{\prime}}=x_{\mathrm{S}^{\prime}}+1$. That is, $x_{3}$ become 163 .

For second, $x=(162,164,161,158,156,156,157)$, including three overlapped pixels (162, $164,161)$ of former pixel, and secret bits $\delta=(001)$ are given.

First, we generate the codeword $p$ from the vector $x$ using Eq. (8) and calculate both $S$ and $S$ ' in the same way in the first case. Since $S$ ' is less than or equal to 7, it is belong to the rule of Eq. (10). That is, $x_{6}=155$. For third and fourth cases, we can get the same way like Fig. 3(a) and (b).

\subsection{Theoretical Estimation of PSNR}

The AMSE of SHk_mDH is estimated as follows. Consider a codeword $C_{i}$ of length $n$ $\left(=2^{k}-1\right)$, where we could embed $k$ secret bits. Because the proposed SHk $\_m D H$ embeds every $k$ secret bits with $m$ overlapped pixels recursively. Therefore, embedding secret bits in the previous codeword $C_{i-1}$ and the next codeword $C_{i+1}$ may modify the pixels in the current codeword $C_{i}$.

Originally, when the modification places in overlapped area of are the same, we should modify other two modification places to avoid the collision. There are $j$ modifications, $0 \leq j \leq 3$, in $C_{i}$.

Case (1) $j=0$ :

$A M S E_{0}=\overbrace{\left(\frac{n-m+1}{n+1} \times \frac{1}{n+1} \times \frac{n-m+1}{n+1}\right) \times 0^{2} / n}^{0 \text { modification in } C_{C_{-1}}, C_{i} \text { and } C_{i+1}}$.

Case (2) $j=1$ :

$A M S E_{1}=\overbrace{\left(\frac{n-m+1}{n+1} \times \frac{n}{n+1} \times \frac{n-m+1}{n+1}\right) \times 1^{2} / n}^{\begin{array}{c}1 \text { modification in } C_{i} ; 0 \text { modification in ovelapped } \\ \text { area between } C_{i-1} \text { and } C_{i} ; 0 \text { modification } \\ \text { in ovelapped area between } C_{i} \text { and } C_{i+1}\end{array}}+\overbrace{\left(\frac{m}{n+1} \times \frac{1}{n+1} \times \frac{n-m+1}{n+1}\right) \times 1^{2} / n+}^{\begin{array}{c}0 \text { modification in } C_{i} ; 1 \text { modification in ovelapped } \\ \text { area between } C_{i-1} \text { and } C_{i} ; 0 \text { modification in } \\ \text { ovelapped area between } C_{i} \text { and } C_{i+1}\end{array}}$

$$
\overbrace{\left(\frac{n-m+1}{n+1} \times \frac{1}{n+1} \times \frac{m}{n+1}\right) \times 1^{2} / n}^{\begin{array}{c}
0 \text { modification in } C_{i} ; 0 \text { modification in ovelapped } \\
\text { area between } C_{i-1} \text { and } C_{i} ; 1 \text { modification in } \\
\text { ovelapped area between } C_{i} \text { and } C_{i+1}
\end{array}} .
$$

Case (3) $j=2$ : 


$$
\begin{gathered}
A M S E_{2}=\overbrace{\left(\frac{m}{n+1} \times \frac{1}{n+1} \times \frac{m}{n+1}\right) \times\left(1^{2}+1^{2}\right) / n}^{\begin{array}{c}
0 \text { modification in } C_{i} ; 1 \text { modification in ovelapped } \\
\text { area between } C_{i-1} \text { and } C_{i} ; 1 \text { modification } \\
\text { in ovelapped area between } C_{i} \text { and } C_{i+1}
\end{array}}+\overbrace{\left(\begin{array}{c}
\left.\frac{m}{n+1} \times \frac{n}{n+1} \times \frac{n-m+1}{n+1}\right) \times\left(1^{2}+1^{2}\right) / n \\
1 \text { modification in } C_{i} ; 0 \text { modification in ovelapped } \\
\text { area between } C_{i-1} \text { and } C_{i} ; 1 \text { modification in } \\
\text { ovelapped area between } C_{i} \text { and } C_{i+1}
\end{array}\right.}^{\begin{array}{c}
1 \text { modification in } C_{i} ; 1 \text { modification in ovelapped } \\
\text { area between } C_{i-1} \text { and } C_{i} ; 0 \text { modification in } \\
\text { ovelapped area between } C_{i} \text { and } C_{i+1}
\end{array}} \\
+\overbrace{\left(\frac{n-m+1}{n+1} \times \frac{n}{n+1} \times \frac{m}{n+1}\right) \times\left(1^{2}+1^{2}\right) / n}^{n+1}+\overbrace{2 \times\left(\frac{n-m+1}{n+1} \times \frac{1}{n+1} \times \frac{m}{n+1}\right) \times 1^{2} / n}^{\text {collisions occur in the second term and the third term }} / n .
\end{gathered}
$$

Case (4) $j=3$ :

$$
A M S E_{3}=\overbrace{\left(\frac{m}{n+1} \times \frac{n}{n+1} \times \frac{m}{n+1}\right) \times\left(1^{2}+1^{2}+1^{2}\right) / n}^{\begin{array}{c}
1 \text { modification in } C_{i} ; 1 \text { modification in ovelapped } \\
\text { area between } C_{i-1} \text { and } C_{i} ; 1 \text { modification } \\
\text { in ovelapped area between } C_{i} \text { and } C_{i+1}
\end{array}}+\overbrace{2 \times\left(\frac{m}{n+1} \times \frac{1}{n+1} \times \frac{m}{n+1}\right) \times 1^{2} / n}^{\begin{array}{c}
\text { collisions occur in the ovelapped area between } C_{i-1} \text { and } C_{i} \\
\text { and the ovelapped area between } C_{i} \text { and } C_{i+1}
\end{array}} \text {, }
$$

Finally, the AMSE of SH $k \_m D H$ is derived as follows. For simplicity, we may neglect the cases that the collision in overlapped area to get a simple form of AMSE as $\frac{1}{2^{k}}+\frac{m /\left(2^{k}-1\right)}{2^{k-1}}$ (see Eq.(11)).

$$
\left\{\begin{array}{l}
A M S E_{S H k_{-} m}=\sum_{j=0}^{3} A M S E_{j}=\frac{\overbrace{2 m+n}^{\text {no collision }}}{n+1} / n+\overbrace{\frac{2 m \cdot(n-m+2)}{(n+1)^{3}} / n}^{\text {collisions occur }} \\
\approx \frac{2 m+n}{n+1} / n=\frac{2 m+2^{k}-1}{2^{k}} /\left(2^{k}-1\right)=\frac{1}{2^{k}}+\frac{m /\left(2^{k}-1\right)}{2^{k-1}} .
\end{array}\right.
$$

\section{Experiment and Comparison}

\subsection{Experimental Results}

To demonstrate the performance of the proposed method, we compare it with HDH, H1DH, Kim \& Yang's DH, and Hk_mDH by using PSNR and EC. In this experiment, we use $512 \times 512$ original grayscale images as cover images [19]. The PSNR is an expression for the ratio between the maximum possible value (energy) of a signal and the energy of distorting noise that affects the quality of its representation. The visual quality of digital images is very subjective because of personal bias. This is why we should establish quantitative/empirical measures to compare the effects of image quality. Using the same set of tests images, different DH algorithms can be compared fairly to identify which algorithm has better results. If a stego image can closely resemble the original, then it is a good algorithm. In this aspect, the PSNR may help us to do this.

The value of MSE used to measure average the squared intensity differences between a distorted image and reference image. If an image has a small MSE value, it has a relatively good visual quality. The MSE between two image $x$ and $y$ is

$$
\operatorname{MSE}(x, y)=\frac{1}{N} \sum_{i=1}^{N}\left(x_{i}-y_{i}\right)^{2} .
$$

The value $e_{i}=x_{i}-y_{i}$ denotes the difference between the original and distorted signal. The MSE is a function for PSNR measure where $L$ is the dynamic range of allowable pixel intensities. For example, for an 8-bit per pixel image, $L=2^{8}-1=255$. 


$$
P S N R=10 \log _{10} \frac{L^{2}}{M S E}
$$

Meanwhile, the EC adopts to compare the performance of the DH through measuring embedding capacity of the cover image. Obviously, most DHs endeavor to increase EC without degrading the quality of stego image. Eq. (14) implies that EC is the ratio of the number of message bits $(\|\delta\|)$ to the total number of pixels.

$$
\rho=\frac{\|\delta\|}{N \times N}
$$

Table 1 shows the comparison among HDH, H1DH, Kim and Yang's DH, Hk_mDH, and the proposed SHk $\_m D H$ which are HC based DHs. Each ER of those is 0.43, 0.5, 0.54, 0.54, and 0.75 , respectively. The ER of SH $k \_m D H$ is higher than those of other DHs. Moreover, the SHk $m m$ DH is also better than that of Kim and Yang's DH in the aspect of ER and PSNR. The

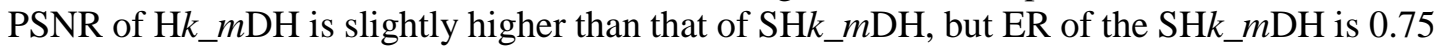
and so it is higher than 0.54 of $\mathrm{H} k \_m D H$.

Table 1. Performance comparison of all Hamming-like DHs

\begin{tabular}{|l|c|c|c|c|c|c|c|c|c|c|}
\hline \multirow{2}{*}{ Images } & \multicolumn{2}{|c}{ HDH } & \multicolumn{2}{c}{ H1DH } & \multicolumn{2}{c|}{ Kim and Yang's } & \multicolumn{2}{c|}{ Hk_mDH } & \multicolumn{2}{c|}{$\begin{array}{c}\text { Sequential } \\
\text { Hk_mDH }\end{array}$} \\
\cline { 2 - 12 } & PSNR & $\rho$ & PSNR & $\rho$ & PSNR & $\rho$ & PSNR & $\rho$ & PSNR & $\rho$ \\
\hline \hline Baboon & 57.1670 & 0.43 & 58.5731 & 0.5 & 53.96 & 0.54 & 56.0045 & 0.54 & 54.2749 & 0.75 \\
\hline Barbara & 57.1598 & 0.43 & 58.5851 & 0.5 & 53.93 & 0.54 & 56.0121 & 0.54 & 54.2874 & 0.75 \\
\hline Boats & 57.1644 & 0.43 & 58.6066 & 0.5 & 53.94 & 0.54 & 55.9930 & 0.54 & 54.2823 & 0.75 \\
\hline Goldhill & 57.1588 & 0.43 & 58.5937 & 0.5 & 53.95 & 0.54 & 56.0005 & 0.54 & 54.2974 & 0.75 \\
\hline Airplane & 57.1497 & 0.43 & 58.5755 & 0.5 & 53.95 & 0.54 & 55.9985 & 0.54 & 54.2943 & 0.75 \\
\hline Lena & 57.1620 & 0.43 & 58.5772 & 0.5 & 53.95 & 0.54 & 56.0164 & 0.54 & 54.3018 & 0.75 \\
\hline Peppers & 57.1687 & 0.43 & 58.5616 & 0.5 & 53.95 & 0.54 & 56.0063 & 0.54 & 54.2832 & 0.75 \\
\hline Tiffany & 57.1780 & 0.43 & 58.6239 & 0.5 & 53.96 & 0.54 & 56.0104 & 0.54 & 54.2949 & 0.75 \\
\hline Zelda & 57.1677 & 0.43 & 58.5806 & 0.5 & 53.99 & 0.54 & 56.0112 & 0.54 & 54.2902 & 0.75 \\
\hline Average & 57.1640 & 0.43 & 58.5863 & 0.5 & 53.95 & 0.54 & 56.0058 & 0.54 & 54.2896 & 0.75 \\
\hline
\end{tabular}

Table 2. Performance of SH $k m$ DH for $1 \leq m \leq 3$.

\begin{tabular}{|c|c|c|c|c|c|c|}
\hline \multirow{2}{*}{ Images } & \multicolumn{2}{|c|}{$m=1$} & \multicolumn{2}{c|}{$m=2$} & \multicolumn{2}{c|}{$m=3$} \\
\cline { 2 - 7 } & PSNR & $\rho$ & PSNR & $\rho$ & PSNR & $\rho$ \\
\hline \hline Baboon & 56.3327 & 0.5 & 55.4245 & 0.6 & 54.2749 & 0.75 \\
\hline Barbara & 56.3379 & 0.5 & 55.4020 & 0.6 & 54.2874 & 0.75 \\
\hline Boats & 56.3391 & 0.5 & 55.4134 & 0.6 & 54.2823 & 0.75 \\
\hline Goldhill & 56.3486 & 0.5 & 55.4075 & 0.6 & 54.2974 & 0.75 \\
\hline Airplane & 56.3399 & 0.5 & 55.3981 & 0.6 & 54.2943 & 0.75 \\
\hline Lena & 56.3422 & 0.5 & 55.3820 & 0.6 & 54.3018 & 0.75 \\
\hline Peppers & 56.3403 & 0.5 & 55.4063 & 0.6 & 54.2832 & 0.75 \\
\hline Tiffany & 56.3469 & 0.5 & 55.4177 & 0.6 & 54.2949 & 0.75 \\
\hline Zelda & 56.3305 & 0.5 & 55.3951 & 0.6 & 54.2902 & 0.75 \\
\hline Average & 56.3397 & 0.5 & 55.4051 & 0.6 & 54.2896 & 0.75 \\
\hline
\end{tabular}


H1DH is superior in quality compared to other DHs, but the proposed SHk_mDH is the highest among all DHs in the respect of PSNR. Especially, SHk_mDH and $\mathrm{H} k \_m D H$ will be able to determine ER using the parameter $m$. In fact, there is a trade-off between PSNR and ER. We may adjust the value of $m$ according to our application.

Table 2 shows the performance of SH $k m D H$ based on variable $m$. For $1 \leq m \leq 3$, ERs are $0.5,0.6$, and 0.75 , respectively. That is to say, ERs increase as increasing the parameter $m$. Meanwhile, PSNRs decrease. The strength of our SHk_mDH is that users may embed a large number of bits by increasing the value of $m$ with retaining the high PSNR.

Table 3. Performance comparison of proposed scheme and previous schemes (when ER =0.34)

\begin{tabular}{|l|c|c|c|c|c|}
\hline \multirow{1}{*}{ Images } & $\begin{array}{c}\mathrm{HDH} \\
(n=7, k=3)\end{array}$ & $\begin{array}{c}\mathrm{H} 1 \mathrm{DH} \\
(n=7, k=3)\end{array}$ & $\begin{array}{c}\text { Kim and Yang’s } \\
\mathrm{DH}(n=7, k=3)\end{array}$ & $\begin{array}{c}\text { Hk_mDH } \\
(n=7, k=3)\end{array}$ & $\begin{array}{c}\text { SHk_mDH } \\
(n=15, k=4)\end{array}$ \\
\cline { 2 - 6 } & PSNR & PSNR & PSNR & PSNR & PSNR \\
\hline \hline Baboon & 58.1147 & 60.2717 & 57.8956 & 57.9809 & 58.9346 \\
\hline Barbara & 58.1150 & 60.2090 & 57.8783 & 57.9931 & 58.9332 \\
\hline Boats & 58.1231 & 60.2636 & 57.8873 & 57.9963 & 58.9249 \\
\hline Goldhill & 58.1094 & 60.2299 & 57.8964 & 58.0172 & 58.9320 \\
\hline Airplane & 58.1130 & 60.2636 & 57.8823 & 58.0117 & 58.9382 \\
\hline Lena & 58.1241 & 60.2412 & 57.8967 & 58.0127 & 58.9290 \\
\hline Peppers & 58.1295 & 60.2439 & 57.9062 & 58.0261 & 58.9316 \\
\hline Tiffany & 58.1819 & 60.2579 & 57.9517 & 58.0209 & 58.9558 \\
\hline Zelda & 58.1186 & 60.2642 & 57.8952 & 58.0008 & 58.9263 \\
\hline Average & 58.1254 & 60.2494 & 57.8988 & 58.0066 & 58.9339 \\
\hline
\end{tabular}

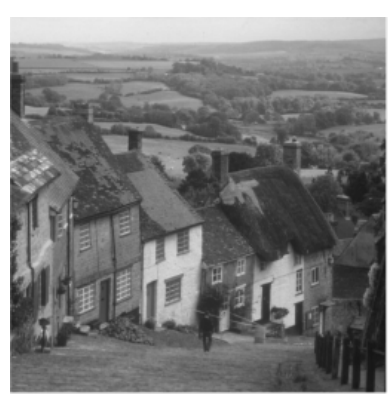

(a)

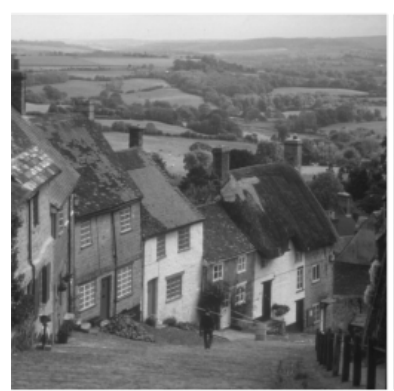

(d)

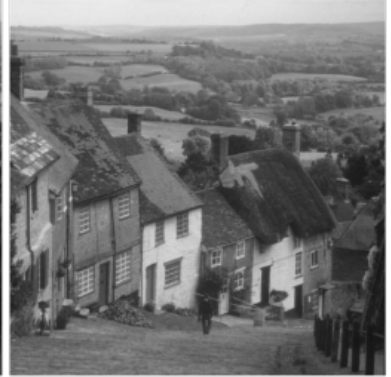

(b)

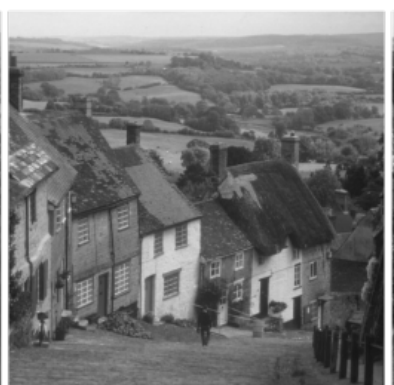

(e)

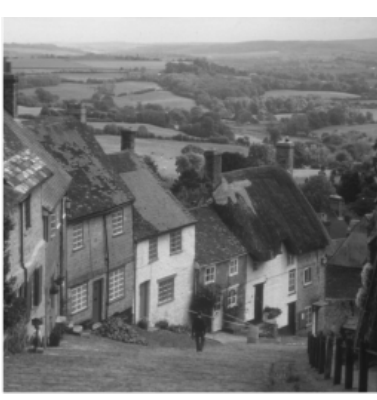

(c)

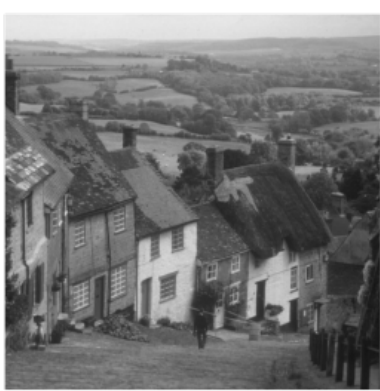

(f)

Fig. 4. Comparison of Goldhill images generated from various schemes: (a) original (b) HDH (58.12 dB), (c) H1DH (60.24 dB), (d) Kim and Yang's DH (57.89 dB), (e) Hk_mDH (58.01 dB), and (f) $\mathrm{SH} \_m \mathrm{mDH}(58.92 \mathrm{~dB})$. 
Table 3 shows the comparison of PSNR between proposed SH $\_m D H$ and previous schemes such as HDH, H1DH, Kim \& Yang's DH, and Hk_mDH when ER is 0.34. As shown in Table 3, even if the same amount of data are hidden in given images, the PSNR of the stego images appears different results depending on the performance of the algorithm. In this experiment, SHk_mDH (in case of COV $(15,4)$ ) had higher PSNRs than HDH, Kim and Yang's DH, and Hk_mDH, while PSNR of H1DH is $0.13 \mathrm{~dB}$ higher than that of SHk_mDH. In fact, SHk_mDH maximize ER while maintaining PSNR.

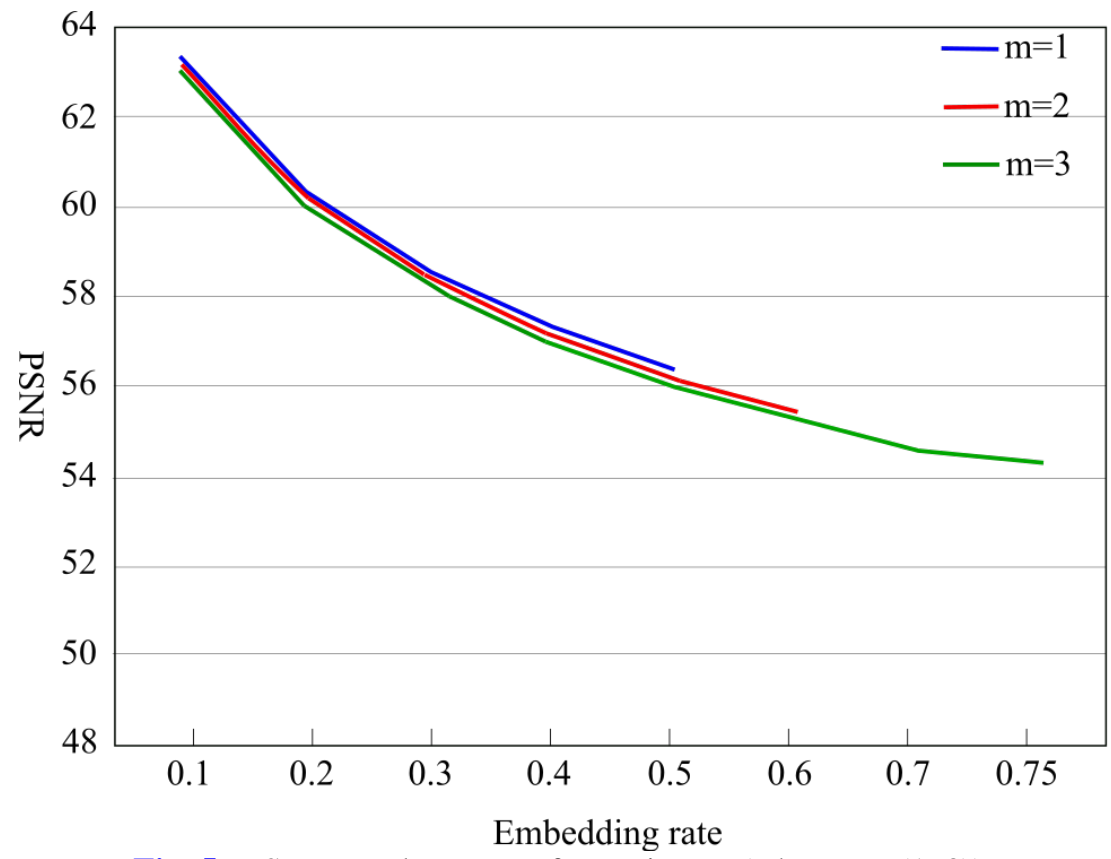

Fig. 5. PSNR trends curves of Lena image (when $m=(1 . .3)$ ).

When ER $=0.34$, Fig. 4 represents the comparison among original images and stego images such as (a) original (b) $\mathrm{HDH}$, (c) H1DH, (d) Kim and Yang's DH, (e) Hk_mDH, and (f) SH $k \_m D H$. The PSNR of H1DH is the highest. The PSNR of SH $\_m D H$ is higher $0.92 \mathrm{~dB}$ than that of $\mathrm{H} k \_m \mathrm{mH}$.

As shown in Fig. 5, experiments were conducted to verify the performance of the proposed method for $1 \leq m \leq 3$ and the ER was (0.1...0.75). The grayscale Lena image is used for ths experiment. For the same ER, the PSNR of $m=1$ is higher than those of $m=2$ and 3.

Entropy [21] is a statistical measure of randomness that can be used to characterize the texture of the input image. The normalized histogram is an estimate of the underlying probability of pixel intensities, i.e., $N / h(i)$, where $h(i)$ denotes the histogram entry of intensity value in an image and $N$ is the total number of pixel. The entropy of an image is computed as:

$$
\mathrm{E}=\sum_{i} h(i) \log \frac{N}{h(i)}
$$

When the relative entropy $\mathbf{E}$ lying between two probability distribution functions is zero, the system is perfectly secure. In Table 4, the entropy of original image (Entropy O), stego image (Entropy S), and the difference between Entropy $\mathrm{O}$ and Entropy $\mathrm{S}$ are enumerated. It appears that when the number of bits in the secret message increases, the relative entropy in stego 
image also increases. Because the differential entropy is approximated to zero, it means the proposed scheme endures an attack of steganalysis tools.

Table 4. Comparison of relative entropy between original images and stego images.

\begin{tabular}{|c|c|c|c|c|}
\hline Image & ER & Entropy O & Entropy S & Difference \\
\hline \hline \multirow{3}{*}{ Lena } & 0.3 & 7.3938 & 7.3937 & 0.0001 \\
\cline { 2 - 5 } & 0.4 & 7.3938 & 7.3939 & 0.0001 \\
\hline \multirow{3}{*}{ Baboon } & 0.3 & 7.3579 & 7.3579 & 0.0000 \\
\cline { 2 - 5 } & 0.4 & 7.3579 & 7.3582 & 0.0003 \\
\hline \multirow{3}{*}{ Boats } & 0.3 & 7.1237 & 7.1256 & 0.0018 \\
\cline { 2 - 5 } & 0.4 & 7.1237 & 7.1257 & 0.0020 \\
\hline \multirow{3}{*}{ Goldhill } & 0.3 & 7.4777 & 7.4794 & 0.0016 \\
\cline { 2 - 5 } & 0.4 & 7.4777 & 7.4791 & 0.0013 \\
\hline \multirow{3}{*}{ Tiffany } & 0.3 & 6.6055 & 6.6064 & 0.0008 \\
\cline { 2 - 5 } & 0.4 & 6.6055 & 6.6066 & 0.0010 \\
\hline
\end{tabular}

RS steganalysis [22] was developed with the intent to detect embedded secret messages using LSB replacement. For RS steganalysis, the discrimination function (DF) $f$ was used (Eq.(16)), which is to capture the smoothness or "regularity" of the group of pixels.

$$
f\left(x_{1}, x_{2}, \ldots, x_{n}\right)=\sum_{i=1}^{n-1}\left|x_{i+1}-x_{i}\right|
$$

A pixel groups can be classified as three types: R, S, and U.

- $\quad$ Regular groups: $\mathrm{G} \in \mathrm{R} \Leftrightarrow f(\mathrm{~F}(\mathrm{G}))>f(\mathrm{G})$.

- Singular groups: $G \in S \Leftrightarrow f(F(G))<f(G)$.

- $\quad$ Regular groups: $G \in U \Leftrightarrow f(F(G))=f(G)$.

where $\mathrm{F}(\mathrm{G})$ is $\left(\mathrm{F}_{\mathrm{M}(1)}\left(x_{1}\right), \mathrm{F}_{\mathrm{M}(2)}\left(x_{2}\right), \ldots, \mathrm{F}_{\mathrm{M}(n)}\left(x_{n}\right)\right)$. Thus, total number of $\mathrm{R}$ groups will be increase than the total number of $S$ groups. When the parameters satisfy Eq.(17), it indicates that there is no hidden data in the respective image. When an image has hidden data, $\mathrm{R}_{-M}$ and $S_{M}$ increases, whereas $R_{M}$ and $S_{M}$ decrease and detected by RS steganalysis.

$$
R_{M} \approx R_{-M} \text { and } S_{M} \approx S_{-M}
$$

According to increase ER, the difference between $R_{M}$ and $S_{M}$ is to zero. After flipping the LSB of about $43 \%$ of pixels, it become $R_{M} \neq S_{M}$, where $M=\left[\begin{array}{lllll}1 & 1 & 0 & 0 & 1\end{array}\right]$. As shown in Fig. 6 (a), it is a few to find the $\mathrm{R}_{M} \approx \mathrm{R}_{-_{M}}$ and $\mathrm{S}_{M} \approx \mathrm{S}_{-\mathrm{M}}$. Meanwhile, in Fig. 6 (b),(c), and (d), we can see $\mathrm{R}_{M}$ $\approx \mathrm{R}_{-\mathrm{M}}$ and $\mathrm{S}_{\mathrm{M}} \approx \mathrm{S}_{-\mathrm{M}}$ until about $100 \%$. Therefore, HDH, Hk_mDH, and SHk_mDH have safety zones avoiding steganalysis detection. 


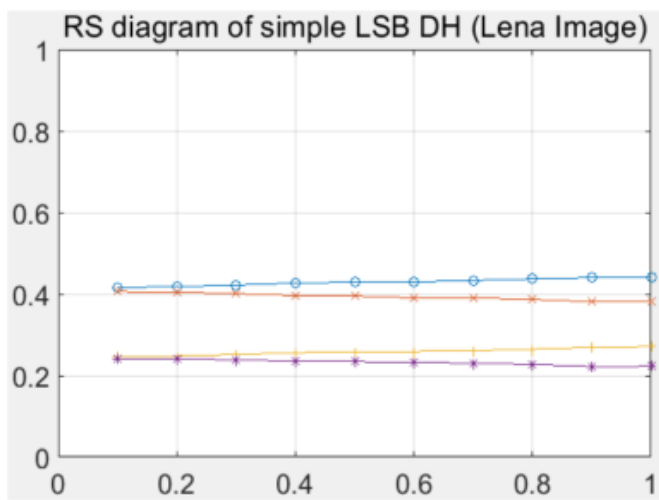

(a)

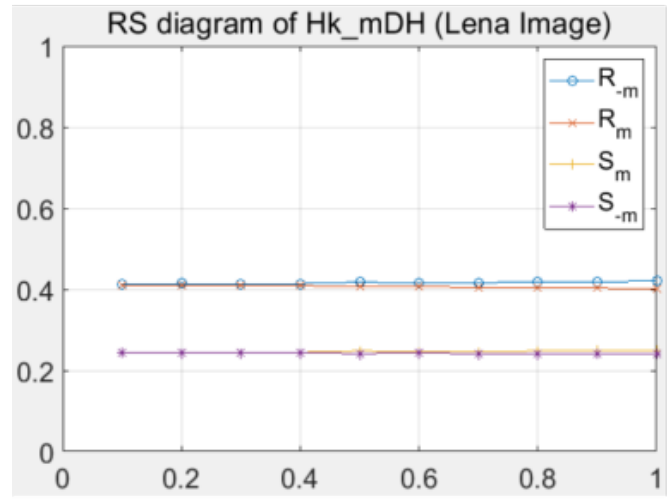

(c)

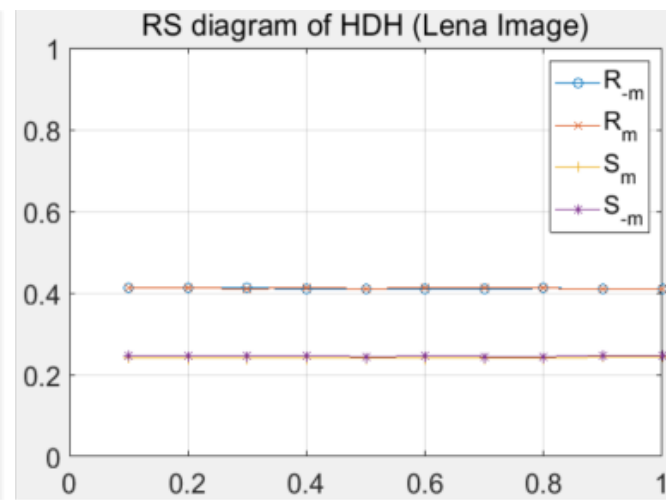

(b)

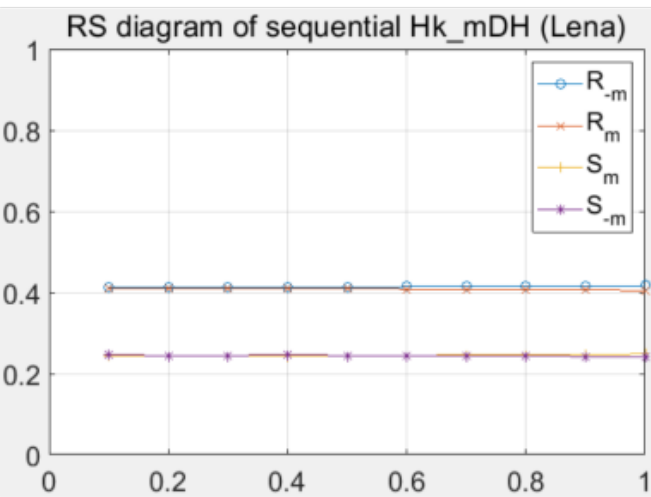

(d)

Fig. 6. Comparison of RS diagram between schemes (when $\mathrm{ER}=0.43$ )

\section{Conclusion}

Hk_mDH is better than previous HC based DHs in the aspect of ER. In this paper, the reason why we proposed SHk $\_m D H$ was that $H k \_m D H$ is not applied sequentially. As a result, could the sequential approach increases the ER to 0.75 when $m=3$. In SHk $m \mathrm{mDH}$, a collision may occur when the same positions in two overlapping blocks need to be corrected at the same time. In this paper, we solve the collision problem using OPAP and LSB. In addition, we demonstrated the superiority of SHk_mDH via experiments and proofs.

\section{Acknowledgements}

This research was supported in part by Ministry of Science and Technology (MOST), under Grant 107-2221-E-259-007-. This research was supported by the Basic Science Research Program through the National Research Foundation of Korea (NRF) funded by the Ministry of Education (2018R1D1A1B07047395), National Research Foundation of Korea (NRF) funded by (2015R1D1A1A01059253), and was supported under the framework of international cooperation program managed by NRF (2016K2A9A2A05005255). 


\section{References}

[1] P. Moulin and R. Koetter, "Data-hiding codes," in Proc. of IEEE, vol. 93, pp. 2083-2126, 2005. Article (CrossRef Link).

[2] W. Bender, D. Gruhl, N. Morimoto, A. Lu, "Techniques for data hiding," IBM Systems Journal, vol. 35, no. 3.4, pp. 313 - 336, 1996. Article (CrossRef Link).

[3] J. Fridrich, M. Goljan, D. Hogea, "Steganalysis of JPEG Images: Breaking the F5 Algorithm," in Proc. of Petitcolas F.A.P. (eds) Information Hiding. IH 2002. Lecture Notes in Computer Science, vol. 2578. Springer, Berlin, Heidelberg, pp.310-323, 2003. Article (CrossRef Link).

[4] Z. Xia, X. Wang, X. Sun, Q. Liu, N. Xiong, "Steganalysis of LSB matching using differences between nonadjacent pixels,” Multimed. Tools Appl., vol. 75, no. 4, pp. 1947-1962, February 2016. Article (CrossRef Link).

[5] C.K. Chan, L.M. Cheng, "Hiding data in images by simple LSB substitution," Pattern Recognition, vol. 37, no. 3, pp. 469-474, March 2004. Article (CrossRef Link).

[6] C.N. Yang, S.C. Hsu, C. Kim, "Improving stego image quality in image interpolation based data hiding," Computer Standards \& Interfaces, vol. 50, pp. 209-215, February 2017. Article (CrossRef Link).

[7] R. Crandall, "Some notes on steganography," 30 November 2018.

[8] A. Westfeld, "F5-A Steganographic Algorithm: High Capacity Despite Better Steganalysis," in Proc. of the 4th International Workshop on Information Hiding, pp. 289-302, 2001. Article (CrossRef Link).

[9] J. Bierbrauer, J. Fridrich, "Constructing good covering codes for applications in steganography," Trans. on Data Hiding and Multimedia Security III, pp. 1-22, 2008. Article (CrossRef Link).

[10] M. van Dijk and F. M. J. Willems, "Embedding information in grayscale images," in Proc. of the 22nd Symposium on Information and Communication Theory in the Benelux, pp. 147-154, (Enschede, The Netherlands), May 2001. Article (CrossRef Link).

[11] W. Zhang, S. Wang, X. Zhang, "Improving embedding efficiency of covering codes for applications in steganography,” IEEE Commun. Lett., vol. 11, pp. 680-682, 2007. Article (CrossRef Link).

[12] R. Zhang, V. Sachnev, B.M. Bakke, H.J. Kim, J. Heo, "An efficient embedder for BCH coding for steganography,” IEEE T. Inform. Theory, vol. 58, no. 12, pp. 7272-7279, December 2012. Article (CrossRef Link).

[13] X. Zhang, S.Wang, "Efficient steganographic embedding by exploiting modification direction," IEEE Communications Letters, vol. 10, no. 11, pp. 781-783, 2006. Article (CrossRef Link).

[14] J. Fridrich, P. Lison`ek, "Grid coloring in steganography," IEEE Transactions on Information Theory, vol. 53, no. 4, pp. 1547-1549, 2007. Article (CrossRef Link).

[15] J. Mielikainen, "LSB matching revisited,” IEEE Signal Proc. Let., vol. 13, pp. 285-287, 2006. Article (CrossRef Link).

[16] W. Zhang, S. Wang, X. Zhang, "Improving embedding efficiency of covering codes for applications in steganography,” IEEE Communications Letters, vol. 11, no. 8, 680-682, 2007. Article (CrossRef Link).

[17] C. Kim, C.N. Yang, "Data hiding based on overlapped pixels using Hamming code," Multimedia Tools Appl., vol. 75, no. 23, pp. 15651-15663, December 2016. Article (CrossRef Link).

[18] C. Kim, D. Shin, C.N. Yang, Y.S. Chou, "Improving capacity of Hamming $(n, k)+1$ stego-code by using optimized Hamming + k,” Digital Signal Processing, vol. 78, pp. 284-293, July 2018. Article (CrossRef Link).

[19] C. Kim, D. Shin, C.N. Yang, Y.S. Chou, "Capacity enhancement of Hamming+k data hiding by pixel overlapping approach," in Proc. of the IEEE 17th International Conference on Communication Technology (ICCT), pp.1242-1246, 2017. Article (CrossRef Link).

[20] "Image database ref.," 31 May 2017. Article (CrossRef Link).

[21] A. Rashid, N. Salamat, V.B.S. Prasath, "An Algorithm for Data Hiding in Radiographic Images and ePHI/R Application,” Technologies, vol. 6, no. 1, pp.1-12, 2018. Article (CrossRef Link). 
[22] J. Fridrich, M. Goljan, R. Du, "Reliable Detection of LSB Steganography in Grayscale and Color Images," in Proc. of the ACM Workshop on Multimedia and Security, Ottawa, Canada, pp. 27-30, October 5, 2001. Article (CrossRef Link).

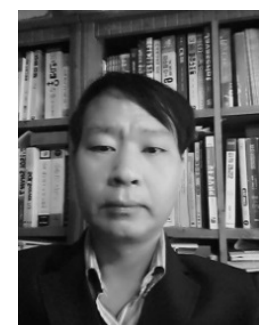

Cheonshik Kim received his B.S. degree in Computer Engineering from Anyang University, Korea, in 1995; his M.S. degree in Computer Engineering from Hankuk University of Foreign Studies (HUFS), Korea, in 1997; and his Ph.D. degree in Computer Engineering from HUFS in 2003. He works at Department of Computer Engineering, Sejong University, Korea. He won a research award from the IEEK in 2012. He is a member of IEEE. His research interests include Multimedia Systems, Data Hiding, and Watermarking. He wrote about 100 papers including conference papers and journal papers. He was a subject of biographical record in the marquis who's who in the world 2013 - 2019.

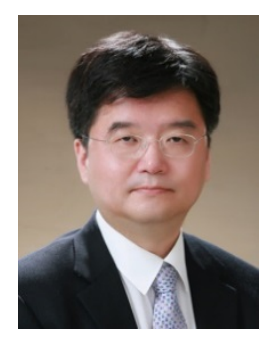

Dongkyoo Shin received a B.S. in Computer Science from Seoul National University, Korea, in 1986, an M.S. in Computer Science from Illinois Institute of Technology, Chicago, Illinois, in 1992, and a Ph.D. in Computer Science from Texas A\&M University, College Station, Texas, in 1997. He is currently an Associate Professor in the Department of Computer Engineering at Sejong University in Korea. From 1986 to 1991, he worked in Korea Institute of Defense Analyses, where he developed database application software. From 1997 to 1998, he worked in the Multimedia Research Institute of Hyundai Electronics Co., Korea as a Principal Researcher. His research interests include Multimedia System, Information Security, Data Mining and Machine Learning.

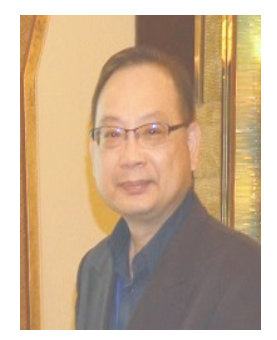

Ching-Nung Yang obtained his Ph. D. degree in Electrical Engineering from National Cheng Kung University. His B.S. and M.S. degrees, both were awarded in Department of Telecommunication Engineering from National Chiao Tung University. Dr. Yang served in National Dong Hwa University since 1999. His current title is Professor in Department of Computer Science and Information Engineering. He had been Visiting Professor to University of Missouri Kansas City, University of Milan, and University of Tokyo. He is currently a Fellow of IET (IEE) and an IEEE senior member. Professor Yang has done extensive researches on visual cryptography and secret image sharing, and is the chief scientist in both areas. He has authored two books and has published over 250 professional research papers (including more than 110 SCI-indexed journal papers) in the areas of information security and coding theory.

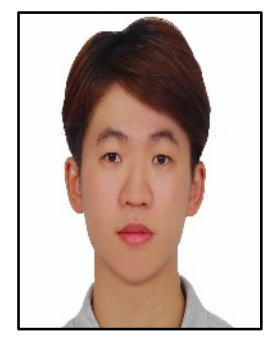

Yi-Cheng Chen is a graduate student at the Department of Computer Science and Information Engineering, National Dong Hwa University, Hualien, Taiwan. His research includes information security and blockchain technology.

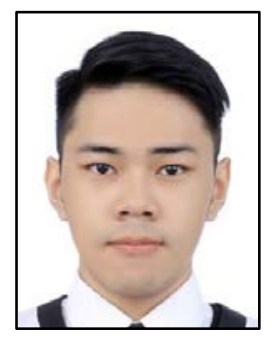

Song-Yu Wu is a graduate student at the Department of Computer Science and Information Engineering, National Dong Hwa University, Hualien, Taiwan. His research includes multimedia security and error correcting code. 